\title{
Bistable electrical switching and performance of a pentacene-based write once/read many memory device
}

\author{
A. G. Gayathri, C. M. Joseph \\ Department of Physics, Dayananda Sagar College of Engg, Shavige malleswara Hills, \\ Kumaraswamy layout, Bangalore-560076, India \\ gaythri305@yahoo.com,cmjoseph@rediffmail.com
}

PACS 72.80.Le, 73.61.Wp, 73.61.Ph, 85.25.Hv

DOI 10.17586/2220-8054-2016-7-4-643-646

\begin{abstract}
In this paper, the performance of a pentacene-based write once/read many memory device is reported. The IV characteristics of a pentacene device deposited at $5 \AA / \mathrm{s}$ on an ITO-coated glass substrate was studied. This device showed a stable switching from ON to OFF state with an ON-OFF current ratio of nearly $10^{3}$ and a retention time of $5 \times 10^{4} \mathrm{~s}$ with a switching threshold voltage of $3.9 \mathrm{~V}$. The irreversible switching of this device makes it suitable for write once/read many memory devices. The structural studies of pentacene thin films on glass substrate were also done and the dependence of device performance on grain size is reported. Improved performance of this device due to the addition of $\mathrm{C}_{60}$ layer is also discussed.
\end{abstract}

Keywords: Organic semiconductor, pentacene, thin films, vacuum thermal evaporation, WORM memory.

Received: 5 February 2016

\section{Introduction}

In recent years, organic memory devices with an active layer sandwiched between two electrodes have attracted much attention as future information storage devices [1-5]. The demand on organic non-volatile memory devices increases rapidly due to their simplicity in device structure, good scalability, low cost potential, low power operation and large capacity for data storage. Electrical bistable switching phenomena have been observed and reported for a variety of organic materials and device structures [1-10]. Several conductance mechanisms are also reported to explain this switching phenomenon.

Different types of organic memory devices are: random access memory, re-writable memory and write once/read many memory (WORM). WORM memory is a type of non volatile memory that is capable of storing data permanently and being read from repeatedly. Thus it is potentially applicable for permanent data storage, such as wireless identification tags, smart cards, etc. Lidan Wang et al. [11] demonstrated a non volatile organic WORM memory device based on $\mathrm{CuPc} / \mathrm{F}_{16} \mathrm{CuPc} \mathrm{p}-\mathrm{n}$ junction. Shengwei Shi et. al. [12] fabricated an organic electrical memory device with a simple structure based on a single layer pentacene film embedded between $\mathrm{Al}$ and ITO electrodes. Bin Li et. al. [13] reported the impact of electrode metals on a pentacene based WORM device. They found that devices with Fe electrodes have lower threshold voltages. However, in terms of application, organic memory devices are often characterized by their performance and materials with good reliability need to be developed.

In this work, we fabricated a write once/read many memory device with vacuum evaporated pentacene on ITO coated glass substrate with aluminium as the top electrode and the performance of the fabricated device was studied and compared with a device with an additional layer of $\mathrm{C}_{60}$.

\section{Experimental}

Figure 1(a) shows the chemical structure of pentacene molecule. Thin films of pentacene $(99.999 \%$ source powder purchased from Aldrich) were thermally deposited onto chemically cleaned glass substrates under vacuum $\left(\sim 2 \times 10^{-6}\right.$ Torr) using a HIND HIVAC coating unit (Model no. 12A4D). For the devices, films were coated on to pre-cleaned ITO coated glass substrates kept at room temperature. Source material was evaporated from a molybdenum boat at a deposition rate of $5 \AA / \mathrm{s}$ to get a thickness of around $90 \mathrm{~nm}$. Finally, aluminium was deposited thermally at a deposition rate of $20 \AA / \mathrm{s}$ from a tungsten basket to complete the device as in Fig. 1(b). The deposition rates and thicknesses of the deposited thin films were controlled by a quartz crystal thickness monitor. The layered structure of the device was Glass/ITO/Pentacene $(5 \AA / \mathrm{s}, 90 \mathrm{~nm}) / \mathrm{Al}(150 \mathrm{~nm})$. For comparison, a layer of $\mathrm{C}_{60}$ was deposited on pentacene layer at a deposition rate of $5 \AA / \mathrm{s}$ to get a thickness of $60 \mathrm{~nm}$. The overlap between the electrodes defined the dimensions of the memory cell $\left(9 \mathrm{~mm}^{2}\right)$. The distance between molybdenum 
source and substrate holder was about $13 \mathrm{~cm}$. The electrical measurements of the fabricated device were measured using a Keithley 2400 source meter and an Agilent B1500A semiconductor device analyzer.

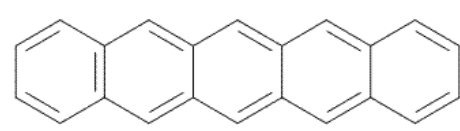

(a)

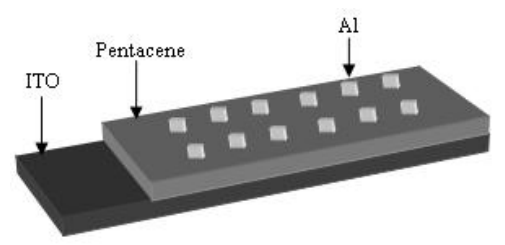

(b)

FIG. 1. Chemical structure of pentacene (a) and schematic view of the fabricated pentacene device (b)

The structural studies were done using a Rigaku X-ray diffractometer. All the electrical tests were conducted at ambient conditions, without any device encapsulation and the measurements were done with a probe dimension of around $14 \mu \mathrm{m}$.

\section{Results and discussion}

\subsection{Structural studies of pentacene thin films}

An XRD spectrum of the pentacene thin films deposited at $5 \AA / s$ is shown in Fig. 2. XRD pattern exhibits crystalline nature with the major diffraction peaks indexed and matches with the previous results [14, 15]. The peak was observed at $2 \theta=5.80$ which corresponds to $\alpha$ phase triclinic pentacene in the (001) plane. Average crystallite size was calculated using Debye-Scherrer formula [16] and was found to be $200 \AA$. The dislocation density was also evaluated using Williamson and Smallman's formula [16] for the deposited films and was found to be $2.5 \times 10^{15} \mathrm{~m}^{-2}$.

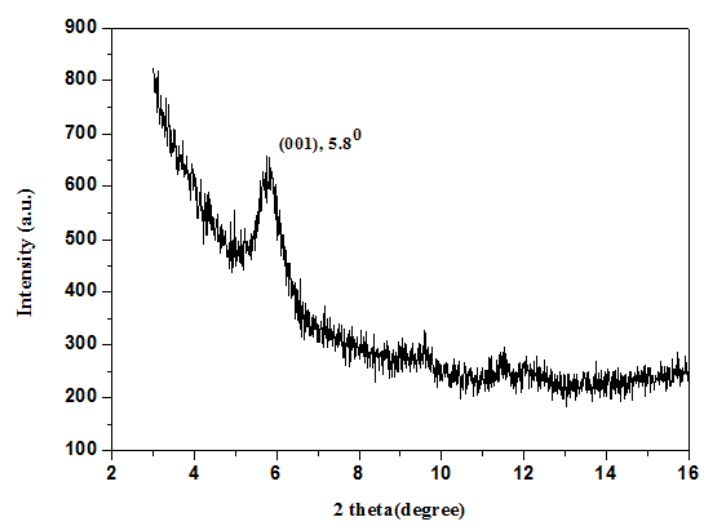

FIG. 2. X-ray diffraction patterns of pentacene thin films grown at $5 \AA / \mathrm{s}$

\subsection{IV characteristics of fabricated device}

Figure 3(a) shows the IV plot of the device ITO/pentacene $(5 \AA / s) / A l$ for a forward and a reverse voltage sweep for 8 continuous voltage sweep cycles. The voltage was scanned from $0 \mathrm{~V}$ to $15 \mathrm{~V}$ and back to $0 \mathrm{~V}$. Initially the device stayed in the high current state which is usually referred to as ON state. At a threshold voltage $V_{T}$ of $3.9 \mathrm{~V}$, the resistance increased suddenly from kiloohms to megaohms, indicating a sudden transition from ON state to OFF state. This hysteresis loop clearly shows the bistability of the device. The ratio of the conductivities achieved between the two states was about $10^{3}$. After this transition, the device remained in the same state even after turning off of the power as shown in the second voltage scan. This revealed the non-volatile nature of this pentacene device. One of the important features of this device was that the switching was irreversible as reported $[12,13]$ and never returned to the initial off state even by applying a negative voltage pulse. This property makes it potentially suitable for a write-once/read many memory (WORM) device. For the ON state, the sweep voltage is between $0 \mathrm{~V}$ and $1.5 \mathrm{~V}$, and the current can be measured very stably at a constant low voltage as long as the current transition has not occurred. For the high resistance state, the sweep voltage is between $0 \mathrm{~V}$ and $15 \mathrm{~V}$. 
After switching to the low current state, the device will stay in the OFF state with the next voltage sweep cycle. The voltage sweep cycles can be more for both ON and OFF states. The stability of the device under stress was evaluated in a continuous bias condition (@1V). The device showed a good performance for $5 \times 10^{4} \mathrm{~s}$ retention time test as shown in the Fig. 3(b).

Figure 4 compares the device performance of the device with and without $\mathrm{C}_{60}$ layer. Addition of $\mathrm{C}_{60}$ layer improved the device performance with an ON-OFF ratio $10^{8}$ and $V_{T}=3.17 \mathrm{~V}$.

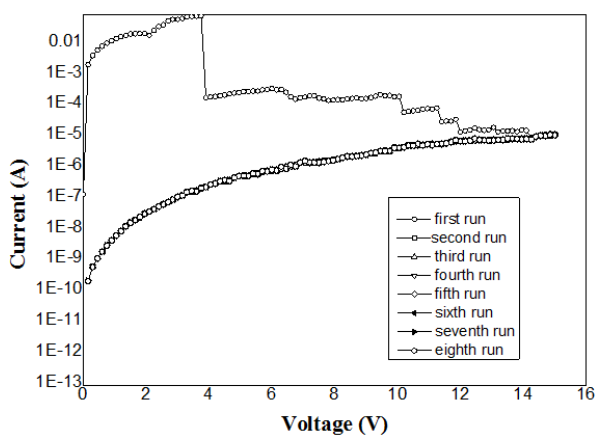

(a)

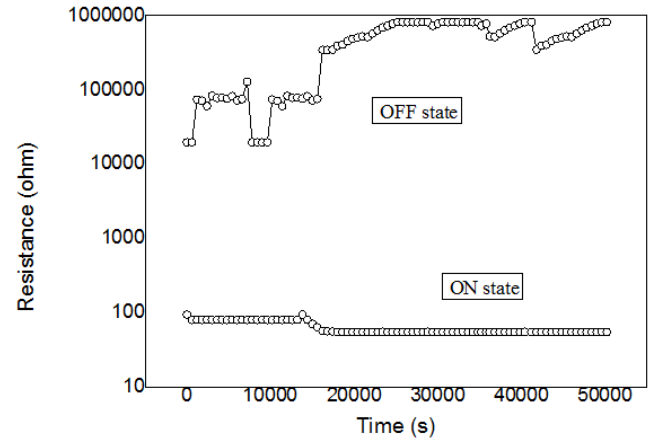

(b)

FIG. 3. (a) IV characteristics of ITO/ pentacene (5 ̊/s)/Al with eight continuous voltage sweep cycles. Inset shows retention time measurements both in the ON state and OFF state (@1V). (b) Retention time measurements both in the ON state and OFF state (@1V).

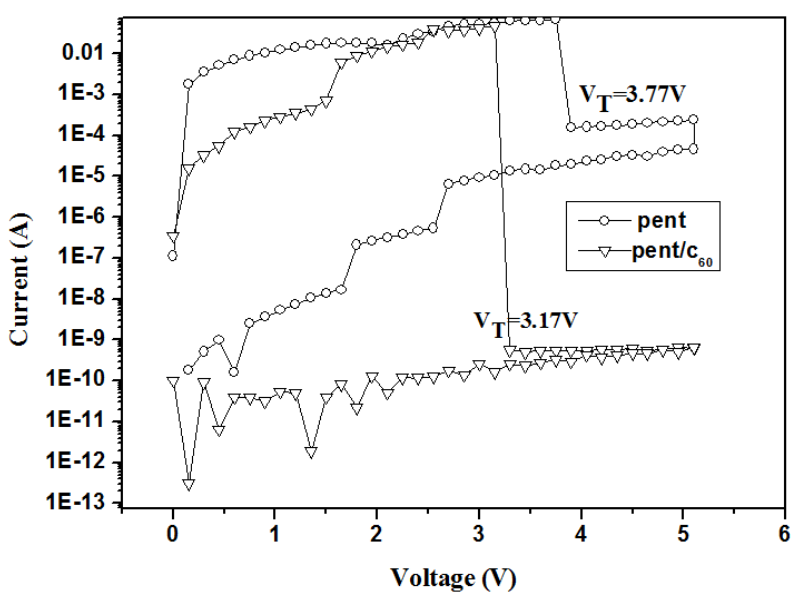

FIG. 4. Comparison of IV characteristics of ITO/Pentacene/Al and ITO/Pentacene/ $\mathrm{C}_{60} / \mathrm{Al}$

The conducting mechanism of a pentacene-based WORM devices were reported earlier [11, 12]. In those devices with a structure $\mathrm{Al} /$ pentacene/ $\mathrm{Al}, \mathrm{Al} /$ pentacene/Fe and $\mathrm{ITO} /$ pentacene/Al, the top metal atom diffuse in to the pentacene layer under positive bias forming conductive channels responsible for high ON state. P. Aruna et al. reported the effect of fullerene doping on the electrical properties of P3HT-PCBM layers [17]. Salaoru I. et al. [6] successfully demonstrated a new donor acceptor small organic molecular pair with tetrathiafulvalene (TTF) as an electron donor and tetracyanoethylene as an electron acceptor element that determined memory behavior. The initial ON state of our devices can be explained on the basis of grain size calculation. The grain size of the films deposited at $5 \AA / \mathrm{s}$ was found to be more. Due to this large grain size, the metal will be diffused into the active layer, which leads to the formation of huge interface dipole layer at the electrode interface resulting in an initial high ON state. However, this interface dipole is destroyed for a large space field resulting in the decrease of the current. Addition of $\mathrm{C}_{60}$ layer significantly improves the device performance due to the molecular storage capability of the fullerenes, significantly enhancing the storage density capability as previously reported [5]. 


\section{Conclusions}

Bistable electrical switching of pentacene devices was studied and compared to that of a device with a $\mathrm{C}_{60}$ layer. The irreversible switching of these devices makes it suitable for a write once read many memory device applications. The devices with pentacene deposited at $5 \AA$ /s showed a stable switching from low resistance state to a high resistance state. This is because as the grain size increases, the penetration of $\mathrm{Al}$ increases, resulting in an initial OFF state. ON-OFF ratio of the films increases from $10^{3}$ to $10^{8}$ with the addition of $\mathrm{C}_{60}$ layer. Threshold voltage decreases from $3.77 \mathrm{~V}$ to $3.17 \mathrm{~V}$ with the addition of $\mathrm{C}_{60}$ layer.

\section{Acknowledgements}

Financial support by Visvesvaraya Technological University (VTU), Belgaum, Karnataka, India through a grant is gratefully acknowledged. Financial support by Vision Group on Science and Technology (VGST), Department of Information Technology, Biotechnology \& Science and Technology, Government of Karnataka, India through a CISE grant is also acknowledged.

\section{References}

[1] Ma L., Xu Q., Yang Y. Organic non-volatile memory by controlling the dynamic copper-ion concentration within organic layer. Appl. Phys. Lett., 2004, 84, P. 4908-4910.

[2] Pyo S., Ma L., et al. Experimental study on thickness related electrical characteristics in organic/metal-nanocluster/organic systems. $J$. Appl. Phys., 2005, 98, P. 054303(1-6).

[3] Ouyong J., Chu C.W., et al. Programmable polymer thin film and non-volatile memory device. Nature materials, 2004, 3, P. 918-922.

[4] Konno K., Sakai H., Matsushima T., Murata H. An organic nonvolatile memory using space charge polarization of a gate dielectric. Thin solid films, 2009, 518, P. 534-536.

[5] Alokik K., Shashi P., Manish C. Organic memory devices using $\mathrm{C}_{60}$ and insulating polymer. Mater. Res. Soc. Symp. Proc., 2005, 830, D7.2.1-D7.2.5.

[6] Salaoru I., Paul S. Memory devices based on small organic molecules donor-acceptor system. Thin Solid Films, 2010, 519, P. 559-562.

[7] Li Y., Chu Y., et al. Synthesis and memory characteristics of polyimides containing noncoplanar aryl pendant groups. Polymer, 2012, 53, P. 229-240.

[8] Thanh Dao T., Matsushima T., Murata H. Organic nonvolatile memory transistors based on fullerene and an electron-trapping polymer. Org. Electronics, 2012, 13, P. 2709-2715.

[9] Min Kim D., Ko Y.G., et al. Digital memory behaviors of aromatic polyimides bearing bis(trifluoromethyl)-and bithiophenyl-triphenylamine units. Polymer, 2012, 53, P. 1703-1710.

[10] Ling Q.D., Liaw D.J., et al. Polymer memories: Bistable electrical switching and device performance. Polymer, 2007, 48, P. $5182-5201$.

[11] Wang L., Su Z., Wang C. Interfacial dipole in organic p/n junction to realize write-once/read-many-times memory. Org. Electronics, 2013, 14, P. 1163-1169.

[12] Shi S., Peng J., Lin J., Ma D. Write-once read-many-times memory based on a single layer of Pentacene. Electron. Dev. Lett., 2009, 30, P. 343-345.

[13] Bin L., Kao C.Y., Arthur J.E. Impact of electrode metals on a pentacene-based write-once read-many memory device. Letter/Synth. Met., 2010, 160, P. 2385-2388.

[14] Puigdollers J., Voz C., et al. Pentacene thin films obtained by thermal evaporation in high vacuum. Thin solid films, 2003, 427, P. $367-370$.

[15] Girtan M., Dabos-Seignon S., Stanculescu A. On morphological, structural and electrical properties of vacuum deposited pentacene thin films. Vacuum, 2009, 83, P. 1159-1163.

[16] Onlaor K., Tunhoo B., et al. Electrical bistable properties of copper phthalocyanine at different deposition rates. Solid-State Electronics, 2012, 72, P. 60-66.

[17] Aruna P., Suresh K., Joseph C.M. Effect of fullerene doping on the electrical properties of P3HT/PCBM layers. Materials Science in Semiconductor Processing, 2015, 36, P. 7-12. 